\title{
Study the Thermal Gradient Effect on Frequencies of a Trapezoidal Plate of Linearly Varying Thickness
}

\author{
Arun Kumar Gupta, Pragati Sharma \\ Department of Mathematics, M. S. College, Saharanpur, India \\ E-mail:gupta_arunnitin@yahoo.co.in,prgt.shrm@gmail.com \\ Received August 12, 2010; revised August 24, 2010; accepted August 27, 2010
}

\begin{abstract}
In this paper, effect of thermal gradient on vibration of trapezoidal plate of varying thickness is studied. Thermal effect and thickness variation is taken as linearly in $\mathrm{x}$-direction. Rayleigh Ritz technique is used to calculate the fundamental frequencies. The frequencies corresponding to the first two modes of vibrations are obtained for a trapezoidal plate for different values of taper constant, thermal gradient and aspect ratio. Results are presented in graphical form.
\end{abstract}

Keywords: Frequencies, Trapezoidal Plate, Linearly Thickness, Thermal Gradient

\section{Introduction}

With the advancement of technology, plates of variable thickness are being extensively used in civil, electronic, mechanical, aerospace and marine engineering applications. It becomes very necessary, now a day, to study the vibration behaviour of plates to avoid resonance excited by internal or external forces. A number of researchers have worked on free vibration analysis of plates of different shapes and variable thickness. Trapezoidal plates are widely used in various structures; however, they have been poorly studied, unlike other plates. Trapezoidal plates find various applications in the construction of modern high speed air craft. The vibration characteristics of such plates are of interest to the designer. Liew and Lam [1] worked on the vibrational response of symmetrically laminated trapezoidal composite plates with point constraints. Chopra and Durvasula [2] worked on the vibration of simply supported trapezoidal plates. Orthotropic plates with clamped boundary conditions were studied by Narita, Maruyama and Sonoda [3]. Liew and Lim [4] have studied the free transverse vibrational analysis of symmetric trapezoidal plates with linearly varying thickness. Qatu, Jaber and Leissa [5] have worked on the natural frequencies of trapezoidal plates with completely free boundaries. Qatu [6] presents the natural frequencies for laminated composite angle-ply triangular and trapezoidal plates with completely free boundaries. Bambill, Laura and Rossi [7] have studied the transverse vibrations of rectangular, trapezoidal and triangular orthotropic cantilever plates. Chen, Kitipornchai, Lim and Liew [8] have worked on the free vibration of cantilevered symmetrically laminated thick trapezoidal plates. Saliba [9-10] discussed the free vibration analysis of simply supported symmetrical trapezoidal plates as well as the transverse free vibration of fully clamped symmetrical trapezoidal plates. Krishnan and Deshpande [11] have studied the free vibration analysis of the trapezoidal plates. Gupta et al. [12-15] have worked on the vibration analysis of visco-elastic rectangular plates of variable thickness and studied the thermal gradient effect and non-homogeneity effect on the free vibrations of the plate. Huang, Hsu and Lin [16] studied the experimental and numerical investigations for the free vibration of cantilever trapezoidal plates. Tomar and Gupta [17-18] studied the effect of thermal gradient on frequencies of orthotropic rectangular plate of variable thickness in one and two direction.

As till now no authors discussed the thermally induced vibration of trapezoidal plate of variable thickness. So, in this paper the temperature effect on vibration of trapezoidal plate of linearly varying thickness is studied. Free transverse vibrations of trapezoidal plates of varying thickness with two opposite simply supported edges have been studied on the basis of classical plate theory. In order to calculate natural frequencies for first and second mode of vibration, Rayleigh Ritz method is used. The frequencies for the first and second mode of vibration is calculated for the trapezoidal plate having C-S-C-S edges for the different values of taper constant, thermal gradi- 
ent and aspect ratio and presented in graphically form.

\section{Method of Analysis \& Equation of Motion}

A symmetric trapezoidal plate, as shown in Figure 1, of varying thickness is taken into consideration. The thickness of the plate $h(\xi)$ is linear in $x$ direction and is of

the form [4]: $h(\xi)=h_{0}\left[1-(1-\alpha)\left(\xi+\frac{1}{2}\right)\right]$

where $h_{0}$ is the maximum plate thickness occurs at left edge $\& \alpha h_{0}$ is the minimum plate thickness occurring at the right edge, $\alpha$ is the taper constant.

For most of the elastic materials, modulus of elasticity (as a function of temperature) is described [19] as

$$
E=E_{0}(1-\gamma \tau)
$$

where $E_{0}$ is the value of young's modulus along the reference temperature, i.e., at $\tau=0$ and $\gamma$ is the slope of the variation of $E$ with $\gamma$.

Assuming that the temperature of the trapezoidal plates varies linearly in $x$ direction only and if $\tau$ and $\tau_{0}$ denote the increase in temperature above the reference at any point at distance $\xi=\frac{x}{a}$ and at the end $\xi=-\frac{1}{2}$ respectively, then $\tau$ can be expressed as

$$
\tau=\tau_{0}\left(\frac{1}{2}-\xi\right)
$$

where $\xi=\frac{x}{a}$

On substituting value of $\tau$ from (3) into (2), one get

$$
E=E_{0}\left(1-\beta\left(\frac{1}{2}-\xi\right)\right)
$$

where $\beta=\gamma \tau_{0} \quad(0 \leq \beta \leq 1)$, known as thermal gradient.

The expression for kinetic energy $V$ and strain energy $\mathrm{T}$ as given by [4] are:

$$
V=\frac{a b}{2} \int_{A} D(\xi)\left\{\begin{array}{l}
\left(\frac{1}{a^{2}} \frac{\partial^{2} w}{\partial \xi^{2}}+\frac{1}{b^{2}} \frac{\partial^{2} w}{\partial \eta^{2}}\right)^{2} \\
{\left[\begin{array}{l}
\frac{1}{a^{2} b^{2}} \frac{\partial^{2} w}{\partial \xi^{2}} \frac{\partial^{2} w}{\partial \eta^{2}} \\
-(1-v)\left[\begin{array}{c}
\left.\frac{1}{a b} \frac{\partial^{2} w}{\partial \xi \partial \eta}\right)^{2}
\end{array}\right.
\end{array}\right]}
\end{array}\right\} d A
$$

and

$$
\mathrm{T}=\frac{a b}{2} \rho \omega^{2} \int_{A} h(\xi) w^{2} d A
$$

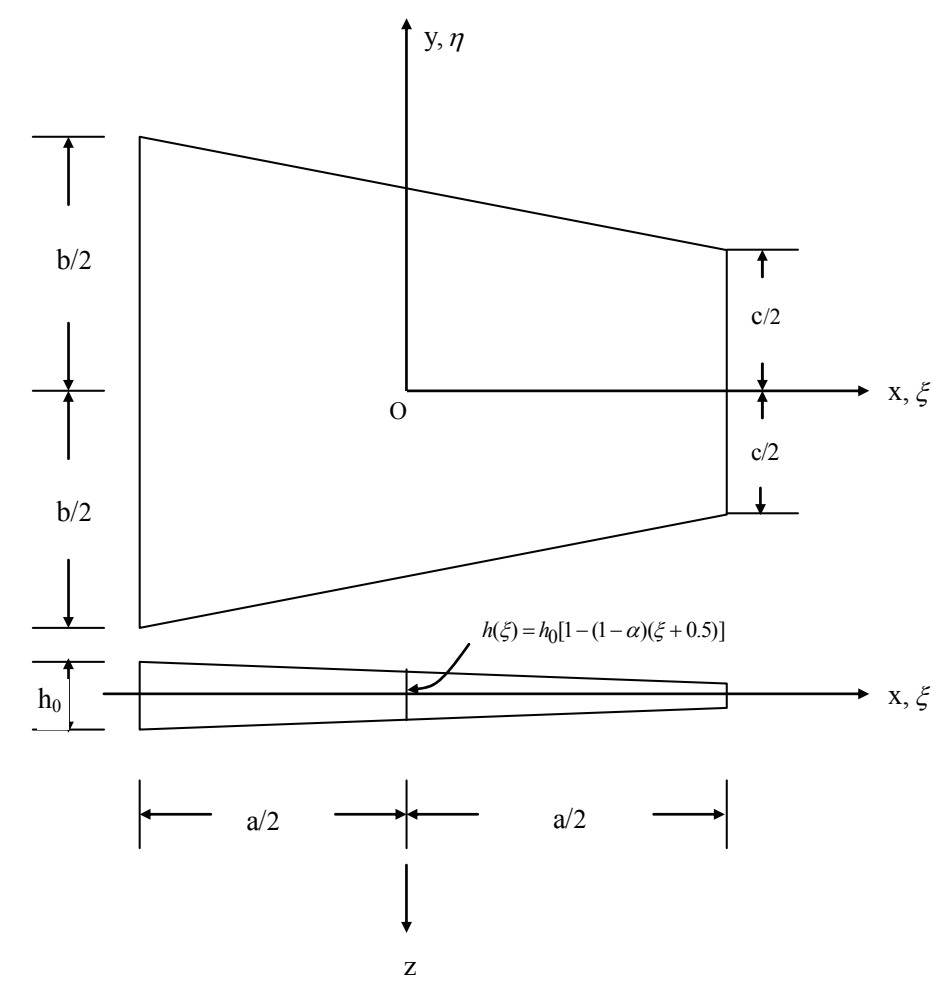

Figure 1. Geometry of trapezoidal plate with variable thickness. 
in which $D(\xi)$ is the flexural rigidity of the plate, which is given by

$$
D(\xi)=D_{0}\left[1-(1-\alpha)\left(\xi+\frac{1}{2}\right)\right]^{3}
$$

$$
\text { where } \quad \xi=\frac{x}{a}, \eta=\frac{y}{b}, D_{0}=\frac{E h_{0}^{3}}{12\left(1-v^{2}\right)}
$$

is the flexural rigidity of the plate, $v$ is the Poisson ratio, $A$ is the area of the plate, $\rho$ is the mass density per unit area of the plate and $\omega$ is the angular frequency of vibration.

Using (8) and (4) in (7)

$$
\begin{aligned}
& D(\xi)=\left[\frac{E_{0} h_{0}^{3}}{12\left(1-v^{2}\right)}\left[1-(1-\alpha)\left(\xi+\frac{1}{2}\right)\right]^{3}\right. \\
& \left.\left[1-\beta\left(\frac{1}{2}-\xi\right)\right]\right]
\end{aligned}
$$

Using (9) in (5)

$$
\begin{aligned}
& V= \\
& \frac{a b}{2} \int_{A} \frac{E_{0} h_{0}^{3}}{12\left(1-v^{2}\right)}\left[1-(1-\alpha)\left(\xi+\frac{1}{2}\right)\right]^{3}\left[1-\beta\left(\frac{1}{2}-\xi\right)\right] \\
& \left.\left\{\begin{array}{l}
\left(\frac{1}{a^{2}} \frac{\partial^{2} w}{\partial \xi^{2}}+\frac{1}{b^{2}} \frac{\partial^{2} w}{\partial \eta^{2}}\right)^{2}- \\
2(1-v)\left[\frac{1}{a^{2} b^{2}} \frac{\partial^{2} w}{\partial \xi^{2}} \frac{\partial^{2} w}{\partial \eta^{2}}-\left(\frac{1}{a b} \frac{\partial^{2} w}{\partial \xi \partial \eta}\right)^{2}\right]
\end{array}\right\}\right] d A
\end{aligned}
$$

Using (1) in (6)

$$
T=\frac{a b}{2} \rho \omega^{2} \int_{A} h_{0}\left[1-(1-\alpha)\left(\xi+\frac{1}{2}\right)\right] w^{2} d A
$$

\section{Solution and Frequency Equations}

Here Rayleigh Ritz technique is used for finding the solution. According to this the maximum strain energy be equal to the maximum kinetic energy i.e.

$$
\delta(V-T)=0
$$

For the plate considered here boundaries are defined by four straight lines

$$
\begin{aligned}
& \eta=\frac{c}{4 b}-\frac{\xi}{2}+\frac{1}{4}+\frac{c \xi}{2 b} \\
& \eta=-\frac{c}{4 b}+\frac{\xi}{2}-\frac{1}{4}-\frac{c \xi}{2 b} \\
& \xi=-\frac{1}{2} \\
& \xi=\frac{1}{2}
\end{aligned}
$$

The two term deflection function taken as,

$$
\begin{aligned}
& w=A_{1}\left\{\left(\xi+\frac{1}{2}\right)\left(\xi-\frac{1}{2}\right)\right\}^{2}\left\{\eta-\left(\frac{b-c}{2}\right) \xi+\frac{b+c}{4}\right\} \\
& \left\{\eta+\left(\frac{b-c}{2}\right) \xi-\frac{b+c}{4}\right\}+A_{2}\left\{\left(\xi+\frac{1}{2}\right)\left(\xi-\frac{1}{2}\right)\right\}^{3} \\
& \left\{\eta-\left(\frac{b-c}{2}\right) \xi+\frac{b+c}{4}\right\}^{2}\left\{\eta+\left(\frac{b-c}{2}\right) \xi-\frac{b+c}{4}\right\}^{2}
\end{aligned}
$$

where $A_{1}$ and $A_{2}$ are constants.

Equation (14) is taken to satisfy boundary condition and provides a good estimation to the frequency.

Two edges of the plates are clamped and two are simply supported i.e. all the four degree of freedoms of the nodes to the side faces of the plates are constrained.

Using (13) in (10) and (11)

$\mathrm{V}=$

$$
\begin{aligned}
& \frac{a b}{2} \int_{-\frac{1}{2}-\frac{c}{4 b}+\frac{\xi}{2}-\frac{1}{4}-\frac{c \xi}{2 b}}^{\frac{1}{4 b}-\frac{\xi}{2}+\frac{1}{4}+\frac{c \xi}{2 b}} \frac{E_{0} h_{0}^{3}}{12\left(1-v^{2}\right)}\left[1-(1-\alpha)\left(\xi+\frac{1}{2}\right)\right]^{3} \\
& {\left[1-\beta\left(\frac{1}{2}-\xi\right)\right]}
\end{aligned}
$$$$
\left\{\begin{array}{l}
\left(\frac{1}{a^{2}} \frac{\partial^{2} w}{\partial \xi^{2}}+\frac{1}{b^{2}} \frac{\partial^{2} w}{\partial \eta^{2}}\right)^{2} \\
-2(1-v)\left[\frac{1}{a^{2} b^{2}} \frac{\partial^{2} w}{\partial \xi^{2}} \frac{\partial^{2} w}{\partial \eta^{2}}-\left(\frac{1}{a b} \frac{\partial^{2} w}{\partial \xi \partial \eta}\right)^{2}\right]
\end{array}\right\} d \eta d \xi
$$

$$
T=\frac{a b}{2} \rho \omega^{2} \int_{-\frac{1}{2}}^{\frac{1}{2}} \int_{-\frac{c}{4 b}+\frac{\xi}{2}-\frac{1}{4}-\frac{c \xi}{2 b}}^{+\frac{c}{4 b}-\frac{\xi}{2}+\frac{1}{4}+\frac{c \xi}{2 b}} h_{0}\left[1-(1-\alpha)\left(\xi+\frac{1}{2}\right)\right] w^{2} d \eta d \xi
$$

Now Equation (12) becomes

$$
\left(V_{1}-\lambda^{2} T_{1}\right)=0
$$


where

$$
\begin{aligned}
& V_{1}=\frac{a b}{2} \int_{-\frac{1}{2}}^{\frac{1}{2}} \int_{-\frac{c}{4 b}+\frac{\xi}{2}-\frac{1}{4}-\frac{c \xi}{2 b}}^{+\frac{c}{4 b}-\frac{\xi}{2}+\frac{1}{4}+\frac{c \xi}{2 b}}\left[1-(1-\alpha)\left(\xi+\frac{1}{2}\right)\right]^{3} \\
& {\left[1-\beta\left(\frac{1}{2}-\xi\right)\right]} \\
& \left\{\begin{array}{l}
\left(\frac{1}{a^{2}} \frac{\partial^{2} w}{\partial \xi^{2}}+\frac{1}{b^{2}} \frac{\partial^{2} w}{\partial \eta^{2}}\right)^{2} \\
-2(1-v)\left[\frac{1}{a^{2} b^{2}} \frac{\partial^{2} w}{\partial \xi^{2}} \frac{\partial^{2} w}{\partial \eta^{2}}-\left(\frac{1}{a b} \frac{\partial^{2} w}{\partial \xi \partial \eta}\right)^{2}\right]
\end{array}\right\} d \eta d \xi \\
& T_{1}=\int_{-\frac{1}{2}}^{\frac{1}{2}} \int_{-\frac{c}{4 b}+\frac{\xi}{2}-\frac{1}{4}-\frac{c \xi}{2 b}}^{+\frac{c}{2}-\frac{\xi}{2}+\frac{1}{4}+\frac{c \xi}{2 b}} h_{0}\left[1-(1-\alpha)\left(\xi+\frac{1}{2}\right) w^{2} d \eta d \xi\right.
\end{aligned}
$$

In Equation (17), $\lambda^{2}=\frac{12 \rho \omega^{2} a^{5}\left(1-v^{2}\right)}{E_{0} h_{0}^{2}}$ is a frequency parameter.

Equation (17) involves the unknown, $A_{1}$ and $A_{2}$ arising due to the substitution of $w$ from Equation (14). These unknowns are to be determined from Equation (17), for which

$$
\left.\begin{array}{c}
\frac{\partial}{\partial A_{1}}\left(V_{1}-\lambda^{2} T_{1}\right)=0 \\
\frac{\partial}{\partial A_{2}}\left(V_{1}-\lambda^{2} T_{1}\right)=0
\end{array}\right\}
$$

On solving (20) we have

$$
b_{m 1} A_{1}+b_{m 2} A_{2}=0
$$

where $b_{m 1}, b_{m 2}(m=1,2)$ involves parametric const. and frequency parameter. The determinant of the coefficient of (21) must vanish for a non-zero solution.

Therefore the frequency equation comes out to be

$$
\left|\begin{array}{ll}
b_{11} & b_{12} \\
b_{21} & b_{22}
\end{array}\right|=0
$$

From Equation (22), a quadratic equation in $\lambda^{2}$ is obtained which gives two values of $\lambda^{2}$.

\section{Result and Discussion}

Frequency (22) is quadratic in $\lambda^{2}$, so it will gives two roots. The frequency is calculated for the first two mode of vibration for a c-s-c-s, trapezoidal plate with linearly varying thickness, for various values of $\mathrm{a} / \mathrm{b}, \mathrm{c} / \mathrm{b}, \beta$, $\alpha$ and $v=.33$. All these results are presented in graphical form. First mode of vibrations is presented in Figure (a) and second mode of vibrations is presented in Figure (b).

Figures 2 and 3: In these figures different values of taper constant $\alpha$ and different aspect ratio $\mathrm{c} / \mathrm{b}=1.0,0.5$ and two values of thermal gradient $\beta=0.0,0.4$ are taken.

Figures 4 and 5: In these figures different values of thermal gradient $\beta$ and different aspect ratio $\mathrm{c} / \mathrm{b}=$ $1.0,0.5, \mathrm{a} / \mathrm{b}=1.0$ and two values of taper constant $\alpha=0.0,0.4$ are taken.

Figures 6 and 7: In these figures different aspect ratio $\mathrm{a} / \mathrm{b}=1.0,0.75, \mathrm{c} / \mathrm{b}=1.0,0.75,0.50,0.25$ and four combination of thermal gradient $\beta$ and taper constant $\alpha$ i.e.

$$
\begin{aligned}
& \alpha=0.0, \beta=0.0 ; \alpha=0.0, \beta=0.4 ; \\
& \alpha=0.4, \beta=0.0 ; \alpha=0.4, \beta=0.4
\end{aligned}
$$

Figures 2 and 3 show that increase in taper constant $\alpha$ makes a increase in frequency for $\beta=0.0,0.4$. Also with increase in aspect ratio $\mathrm{c} / \mathrm{b}$ the frequency decreases for first two mode of vibration.

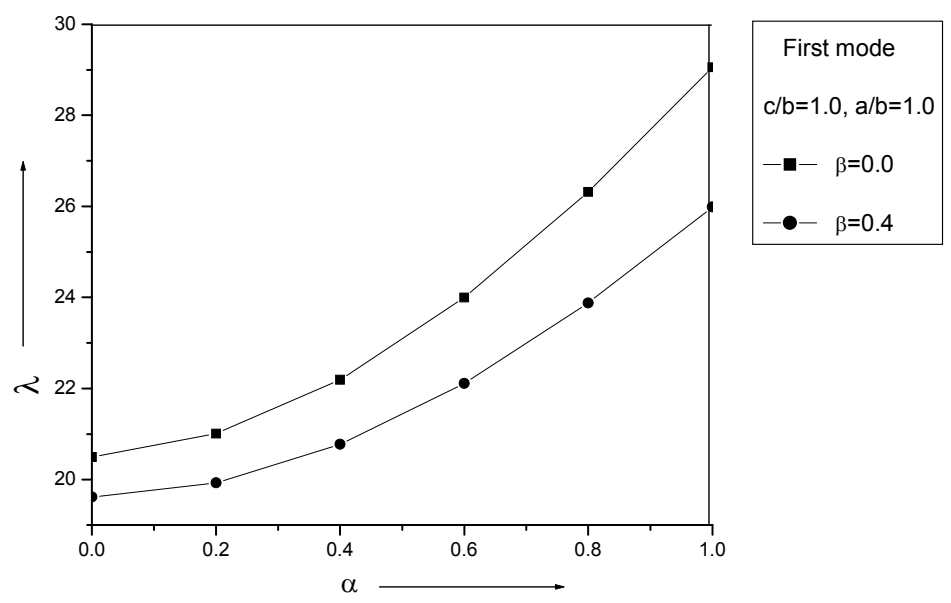

(a) 


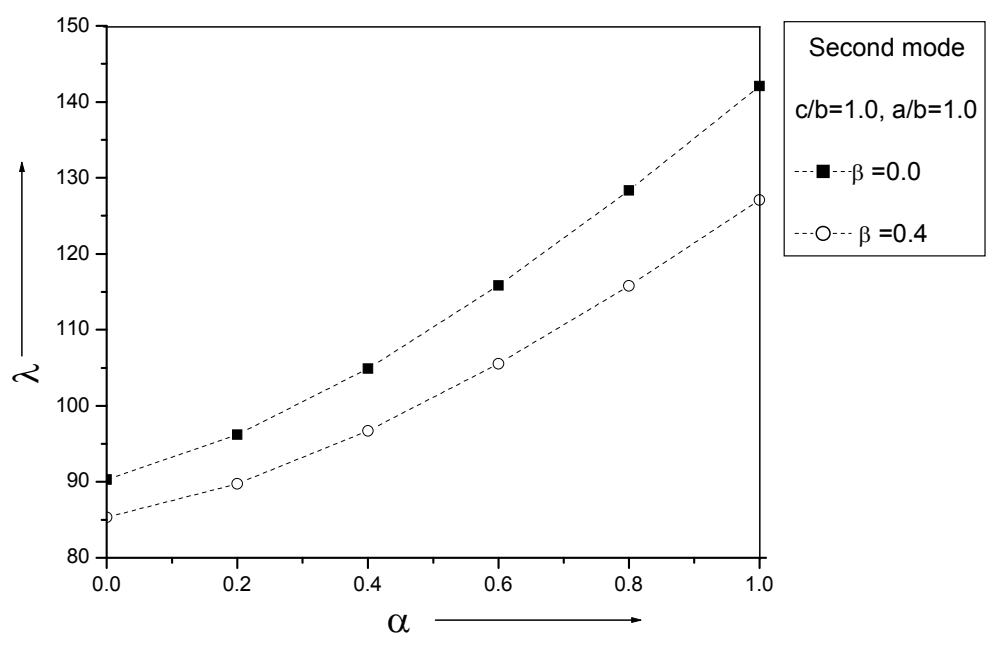

(b)

Figure 2. (a) Frequency parameter $\lambda$ vs. taper constant $\alpha$; (b) Frequency parameter $\lambda$ vs. taper constant $\alpha$.

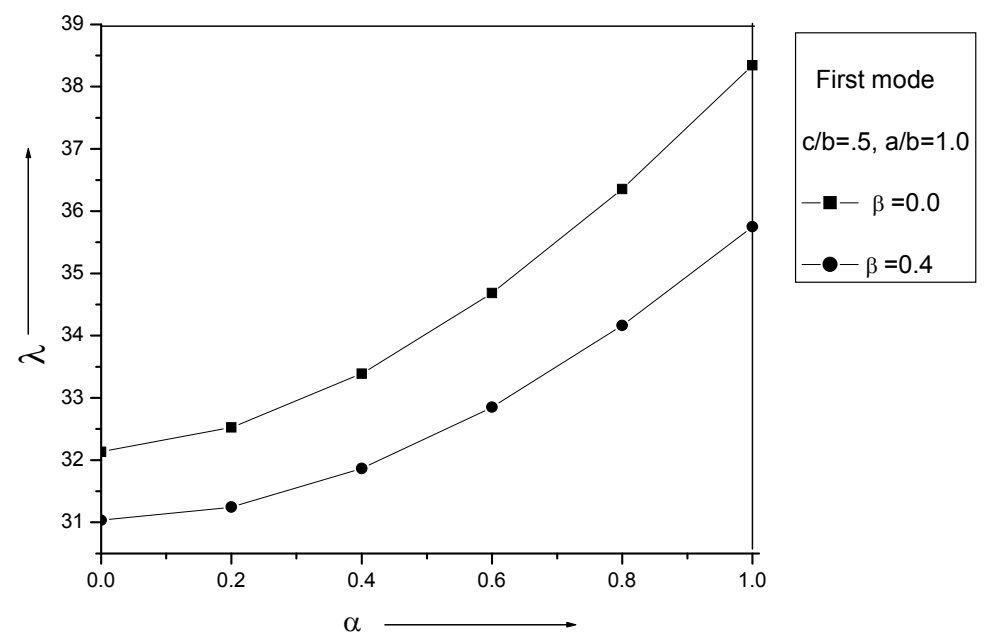

(a)

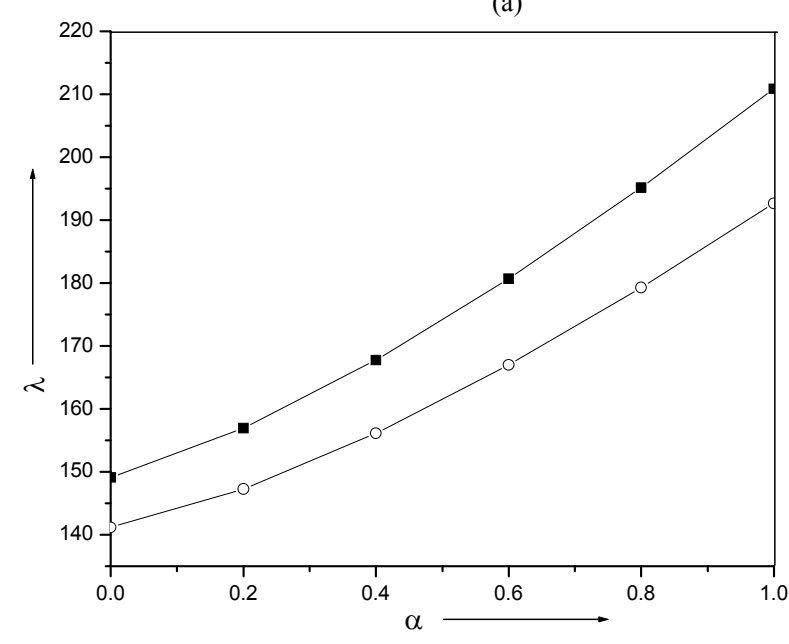

Second mode $\mathrm{c} / \mathrm{b}=.5, \mathrm{a} / \mathrm{b}=1.0$

$-\square-\beta=0.0$

$-O-\beta=0.4$

(b)

Figure 3. (a) Frequency parameter $\lambda$ vs. taper constant $\alpha$; (b) Frequency parameter $\lambda$ vs. taper constant $\alpha$. 

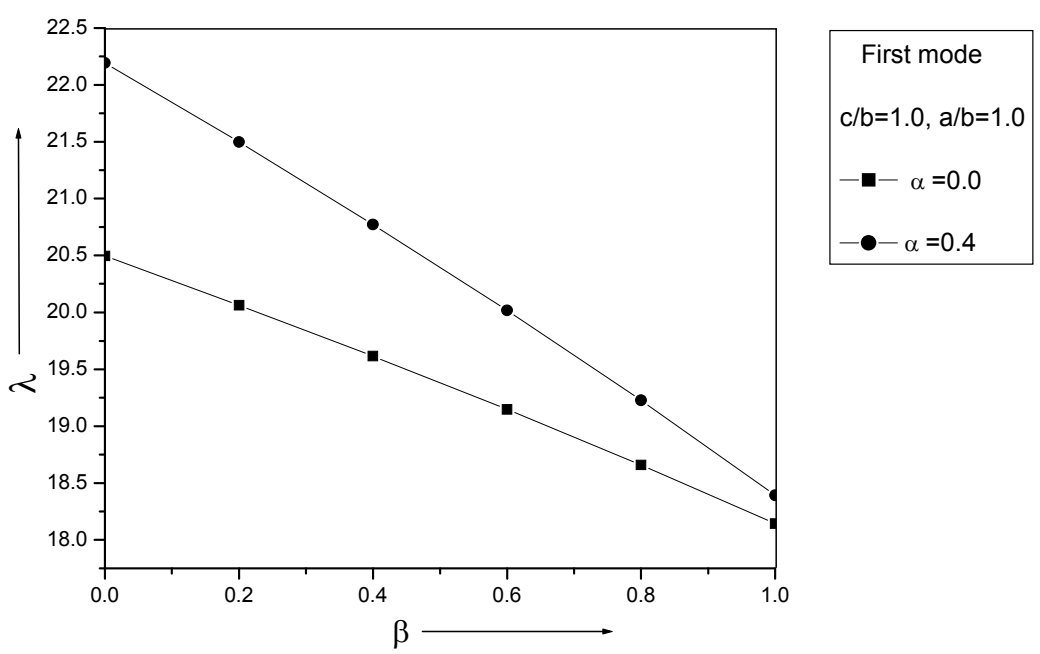

(a)

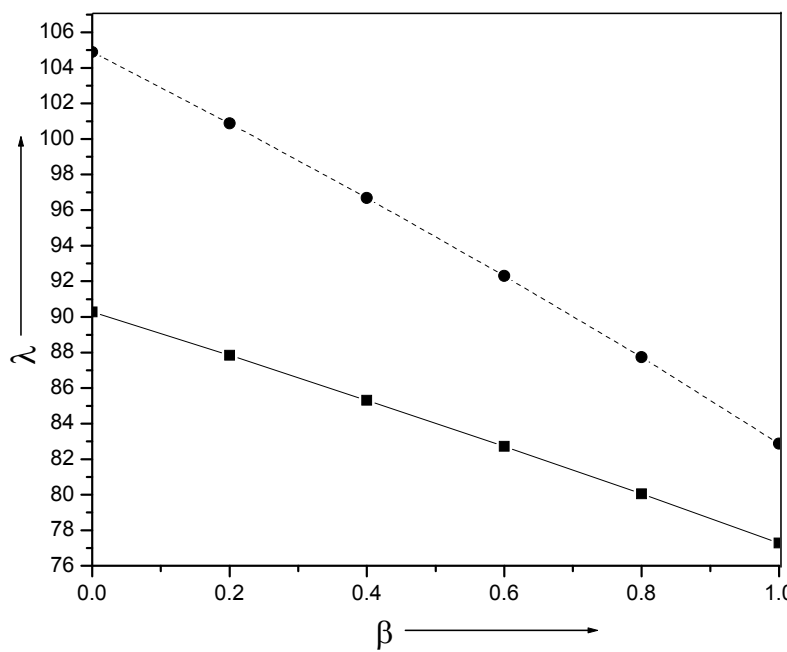

Second mode $c / b=1.0, a / b=1.0$

$-\alpha-\alpha=0$

- $\alpha=0.4$

(b)

Figure 4. (a) Frequency parameter $\lambda$ vs. thermal gradient $\beta$; (b) Frequency parameter $\lambda$ vs. thermal gradient $\beta$.

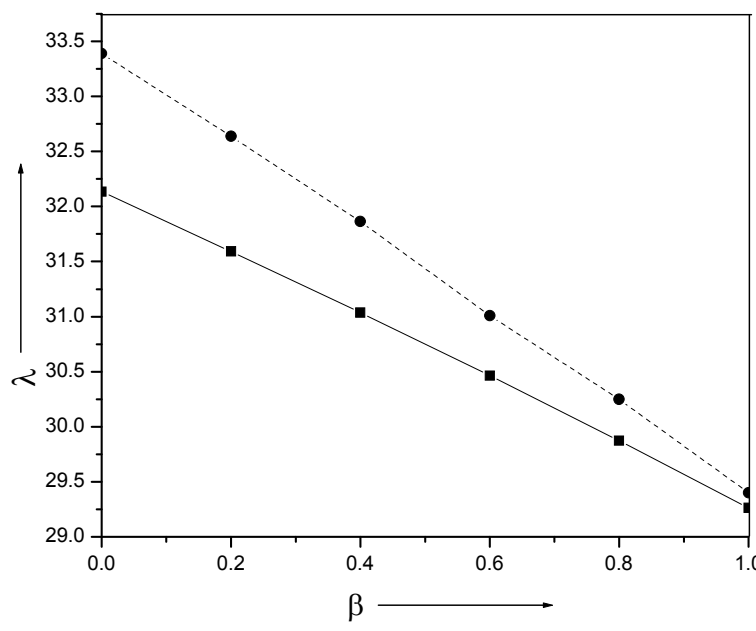

First mode
$\mathrm{c} / \mathrm{b}=0.5, \mathrm{a} / \mathrm{b}=1.0$
$--\alpha=0.0$
$-\alpha=0.4$

(a) 

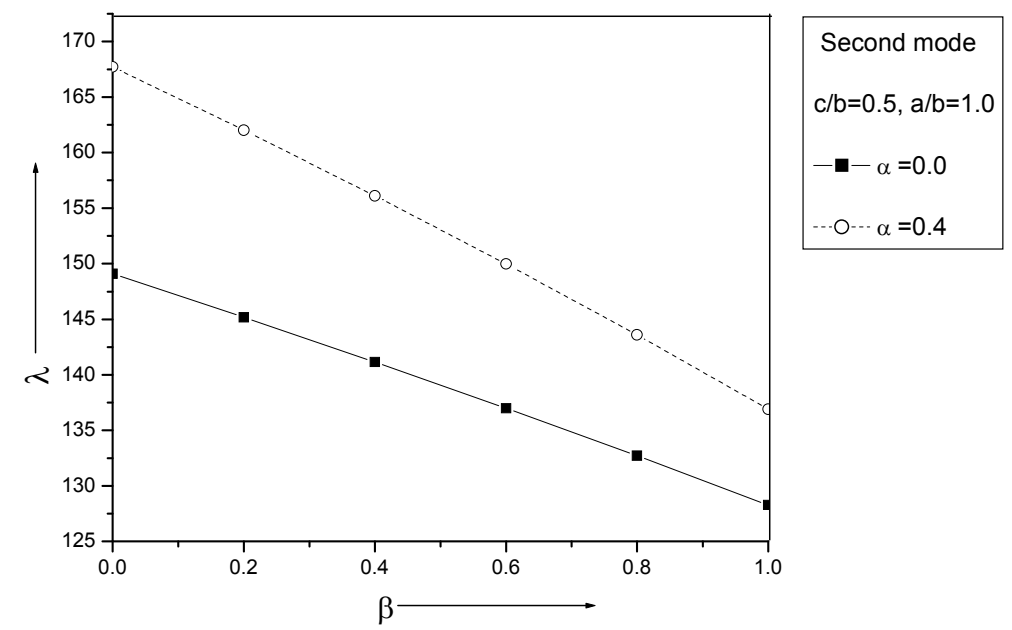

(b)

Figure 5. (a) Frequency parameter $\lambda$ vs. thermal gradient $\beta$; (b) Frequency parameter $\lambda$ vs. thermal gradient $\beta$.
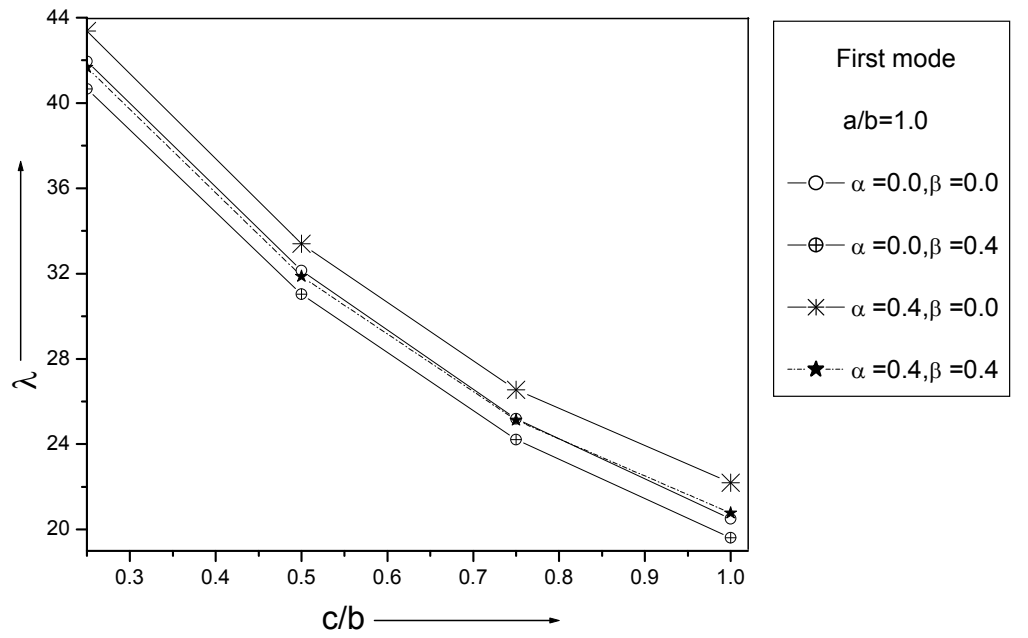

(a)

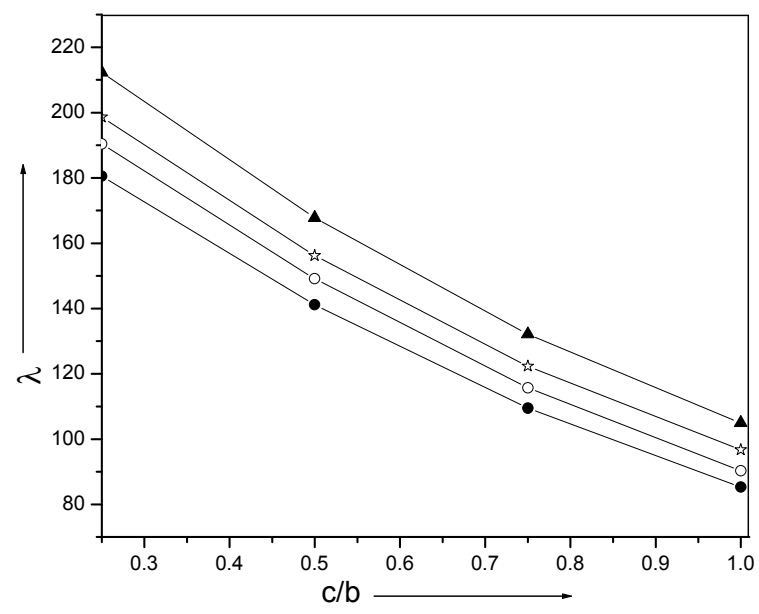

$$
\begin{gathered}
\text { Second mode } \\
\mathrm{a} / \mathrm{b}=1.0 \\
-0-\alpha=0.0, \beta=0.0 \\
-\bullet-\alpha=0.0, \beta=0.4 \\
-\mathbf{\Delta}-\alpha=0.4, \beta=0.0 \\
-\beta-\alpha=0.4, \beta=0.4
\end{gathered}
$$

(b)

Figure 6. (a) Frequency parameter $\lambda$ vs. c/b; (b) Frequency parameter $\lambda$ vs. c/b. 

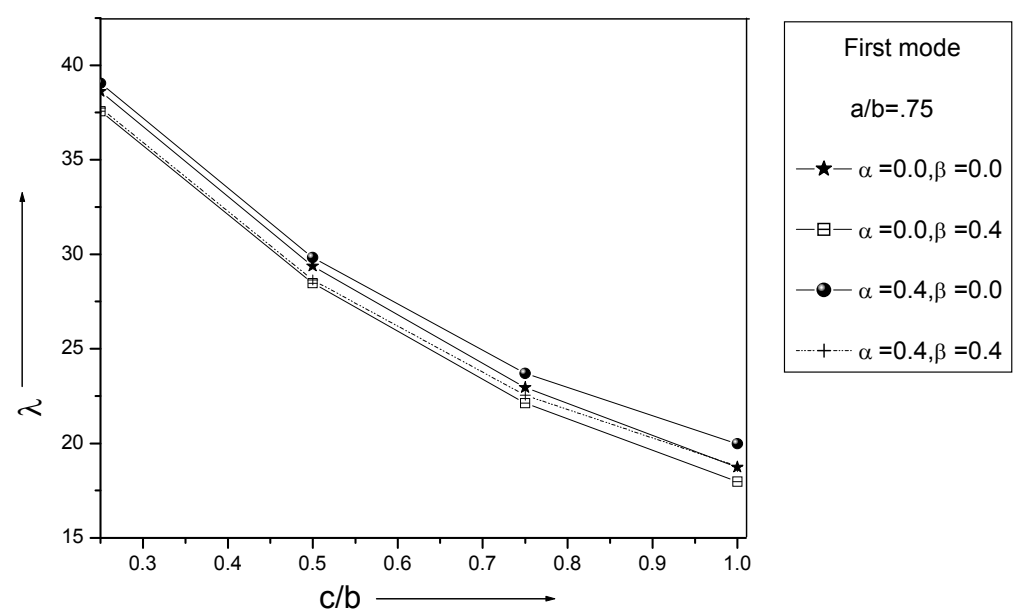

(a)
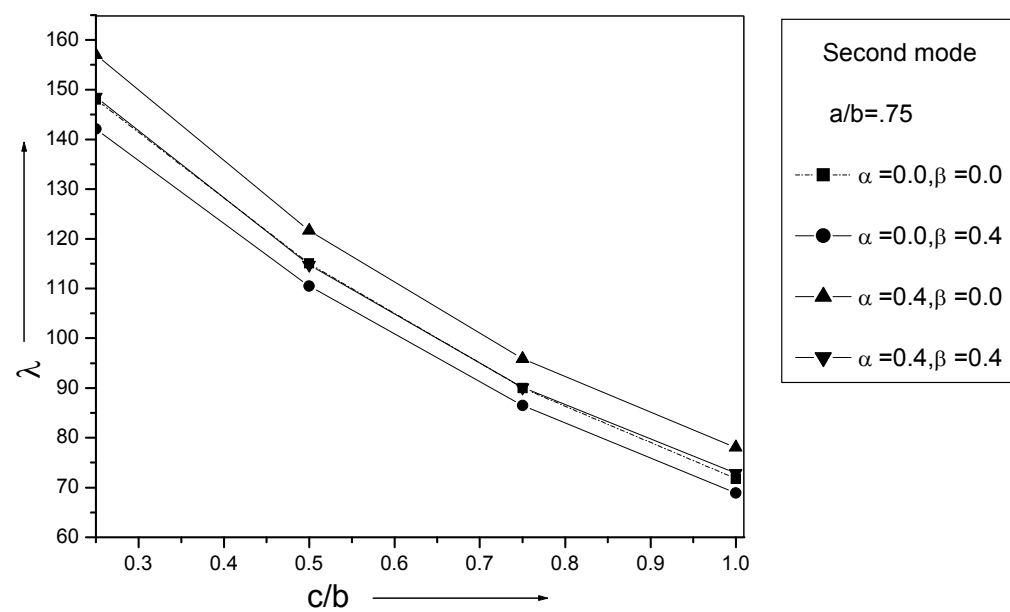

(b)

Figure 7. (a) Frequency parameter $\lambda$ vs. c/b; (b) Frequency parameter $\lambda$ vs. c/b.

Figures 4 and 5 show that increase in thermal gradient $\beta$ the frequency decreases. The result is display- ed for the following two cases of taper constant: $\alpha=0.0,0.4$. Also with increase in aspect ratio $\mathrm{c} / \mathrm{b}$ the frequency decreases for first two mode of vibration.

Figures 6 and 7 show that with increase in aspect ratio $\mathrm{c} / \mathrm{b}$ the frequency decreases for first two mode of vibration. Also with increase in aspect ratio $a / b$ the frequency increase for first two mode of vibration. Also the difference be tween the lines of $\alpha=0.0, \beta=0.0$ and $\alpha=0.4$, $\beta=0.4$ is negligible.

\section{Conclusions}

The Rayliegh-Ritz method has been successfully used to make a comprehensive study of the linear vibration characteristics of trapezoidal plates. Accurate data has obtained by varying the length ratios $a / b$ and $c / b$.
The results for trapezoidal plates of varying thickness are verified by the literature (4). It has been shown that the method provides accurate results.

These results have been determined with considerable accuracy in order that they may serve as future data for researchers who desire to investigate the accuracy of their new numerical methods.

\section{References}

[1] K. M. Liew and K. Y. Lam, "Vibrational Response of Symmetrically Laminated Trapezoidal Composite Plates with Point Constraints," International Journal of Solids and Structures, Vol. 29, No. 24, 1992, pp. 1535-1547.

[2] I. Chopra and S. Durvasula, "Vibration of Simply Supported Trapezoidal Plates I. Symmetric Trapezoids," Journal of Sound Vibration, Vol. 19, No. 4, 1971, pp. 379-392.

[3] Y. Narita, K. Maruyama and M. Sonoda, "Transverse 
Vibration of Clamped Trapezoidal Plates Having Rectangular Orthotropy," Journal of Sound Vibration, Vol. 77, 1981, pp. 345-356.

[4] K. M. Liew and M. K. Lim, "Transverse Vibration of Trapezoidal Plates of Variable Thickness: Symmetric Trapezoids," Journal of Sound Vibration, Vol. 165, No. 1, 1993, pp. 45-67.

[5] M. S. Qatu, N. A. Jaber and A. W. Leissa, "Natural Frequencies for Completely Free Trapezoidal Plates," Journal of Sound Vibration, Vol. 167, No. 1, 1993, pp. 183191.

[6] M. S. Qatu, "Vibrations of Laminated Composite Completely Free Triangular and Trapezoidal Plates," International Journal of Mechanical Sciences, Vol. 36, No. 9, 1994, pp. 797-809.

[7] D. V. Bambill, P. A. A. Laura and R. E. Rossi, "Transverse Vibrations of Rectangular, Trapezoidal and Triangular Orthotropic, Cantilever Plates," Journal of Sound Vibration, Vol. 210, No. 2, 1998, pp. 286-290.

[8] C. C. Chen, S. Kitipornchai, C. W. Lim and K. M. Liew, "Free Vibration of Cantilevered Symmetrically Laminated Thick Trapezoidal Plates," International Journal of Mechanical Sciences, Vol. 41, No. 6, 1999, pp. 685-702.

[9] H. T. Saliba, "Free Vibration Analysis of Simply Supported Symmetrical Trapezoidal Plates," Journal of Sound Vibration, Vol. 110, No. 1, 1986, pp. 87-97.

[10] H. T. Saliba, "Transverse Free Vibration of Fully Clamped Symmetrical Trapezoidal Plates," Journal of Sound Vibration, Vol. 126, No. 2, 1988, pp. 237-247.

[11] A. Krishnan and J. V. Deshpande, "A Study on Free Vibration of Trapezoidal Plates," Journal of Sound Vibration, Vol. 146, No. 2, 1991, pp. 507-515.

[12] A. K. Gupta and H. Kaur, "Study of the Effect of Ther- mal Gradient on Free Vibration of Clamped Visco-Elastic Rectangular Plates with Linearly Thickness Variation in both Directions," Meccanica, Vol. 43, No. 3, 2008, pp. 449-458.

[13] A. K. Gupta, T. Johri and R. P. Vats, "Study of Thermal Gradient Effect on Vibrations of a Non-Homogeneous Orthotropic Rectangular Plate Having Bi-Direction Linearly Thickness Variations," Meccanica, Vol. 45, No. 3, 2010, pp. 393-400.

[14] A. K. Gupta, T. Johri and R. P. Vats, "Thermal Effect on Vibration of Non Homogeneous Orthotropic Rectangular Plate Having Bi-Directional Parabolic Ally Varying Thickness," Proceedings of International Conference on Engineering \& Computer Science, San Francisco, 2007, pp. 784-787.

[15] A. K. Gupta and A. Khanna, "Vibration of Visco-Elastic Rectangular Plate with Linearly Thickness Variations in both Directions," Journal of Sound Vibration, Vol. 301, No. 3-5, 2007, pp. 450-457.

[16] C.-H. Huang, C.-H. Hsu and Y.-K. Lin, "Experimental and Numerical Investigations for the Free Vibration of Cantilever Trapezoidal Plates," Journal of the Chinese Institute of Engineers, Vol. 29, No. 5, 2006, pp. 863-872.

[17] J. S. Tomar and A. K. Gupta, "Effect of Thermal Gradient on Frequencies of an Orthotropic Rectangular Plate Whose Thickness Varies in Two Directions," Journal of Sound Vibration, Vol. 98, No. 2, 1985, pp. 257-262.

[18] J. S. Tomar and A. K. Gupta, "Thermal Effect on Frequencies of an Orthotropic Rectangular Plate of Linearly Varying Thickness," Journal of Sound Vibration, Vol. 90, No. 3, 1983, pp. 325-331.

[19] N. J. Hoff, "High Temperature Effect in Air Craft Structures," Pergamon Press, New York, 1958. 